\title{
Kinect's Opisthenar Vein Image Acquisition Method
}

\author{
Lian Zhang ${ }^{1, a}$,Zixiang Gao $^{2, b}$,Yongxiu Zhou ${ }^{3, c}$
}

${ }^{1}$ Chongqing University of Technology,Banan District Chongqing, China 40054

${ }^{2}$ Chongqing University of Technology,Banan District Chongqing, China 40054

${ }^{3}$ Chongqing University of Technology,Banan District Chongqing, China 40054

azh_lian@cqut.edu.cn, bixiang_gao@126.com, ${ }^{\mathrm{c}} 240499463 @ q q . c o m$

\begin{abstract}
Keywords:Kinect; opisthenar vein; median filter; template matching; ROI
Abstract.Although the current opisthenar vein image acquisition is less demanding, but practical applications often require special equipment, this paper describes a theme based on Microsoft Kinect sensors opisthenar vein acquisition. Using Microsoft's Kinect sensor to collect the opisthenar vein image, introducing correlation-based template matching and ROI two extraction algorithms to get the effective area, after processing, to give a clear opisthenar vein image. Inspection of the acquired image under Matlab environment proves it to be a quick,simple, and easy to implement solution.
\end{abstract}

\section{Introduction}

Venous infrared imaging technology plays an significant role in the section of medical auxiliary and biometric identification, while the vein of hand have more available features of Vein, and compared with the traditional identity recognition, vein recognition contain the advantage of non contact, uniqueness and distinguish living, which is safer than other methods of identification. At the same time, in area of medical, it is more positive to auxiliary venous puncture, existing in the clinical that patients due to the thick adipose layer and other reasons, which led to the low success rate of puncture, therefore, vein imaging technology has been implemented in this field as well. Vein image acquisition occupies more status of work and daily life, but special equipment should be used in general, also exist in specific occasions, for instance: a security place, hospital, etc.

The purpose of this paper, which presents one kind of opisthenar venous collection and solution that based on daily equipment, and enhance the popular speed of venous imaging identification technology. To obtain the grey scale image of opisthenar venous, and then achieve the extracting of effective fields of vein, dealing with the imagination at last. The scheme is simple and easy to achieve, with good adaptability at the same time.

\section{Image Acquisition And Imaging Environment}

Kinect with powerful features is peripherals for the XBOX 360 developed by Microsoft, it is a 3D somatosensory camera, image acquisition mainly includes an infrared projector, infrared CMOS camera, BGB VGA color camera, as shown in Fig.1, the resolutions are $640 \times 480$, and the maximum frame rate of 30fps[1], you can get depth data within the field of view through these three devices, as this article only uses Kinect infrared CMOS camera, other functions and principles will be ignored. It is because Kinect is a game device, which exists in many gaming enthusiasts, making the use of Kinect hand vein image acquisition exists a broad sense, enabling rapid spread of hand vein recognition technology and further development of this technology, also being a simple medical assistive technology.

Due to the low absorption of human skin layer of the infrared spectrum[2], wavelengths between $720 \sim 1100 \mathrm{~nm}$ near infrared can easily penetrate human skin into the subcutaneous tissue[3], so the use of Kinect infrared projector as a light source, an infrared CMOS camera for venous collection is feasible. Use one of the infrared CMOS camera to shoot the opisthenar, while Kinect infrared projector projecting a near-infrared light,whose wavelength is 830nm[4]. 


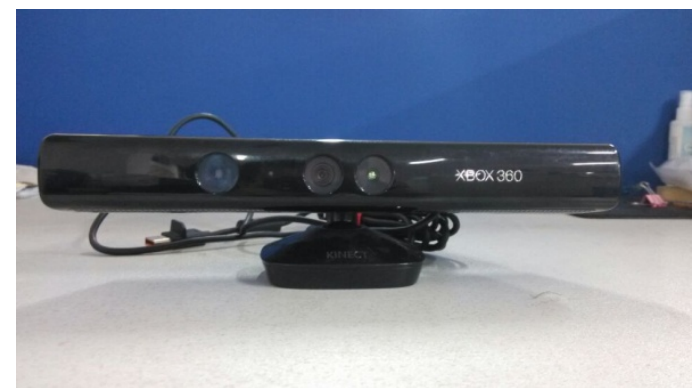

Fig.1 Kinect device for acquiring images

\section{Effective Vein Region Extraction}

To get higher quality of hand vein image, the entire opisthenar image processing is not feasible, which requires locate extract and process of effective hand vein region.

This paper provides two methods to locate extract the hand vein area, one is based on the relevant template matching, and the other is advanced ROI [5] extraction algorithm. Two algorithms will be introduced as follows.

Relevant template matching technology can be directly used to find some kind of template matching subsidiary image mode in an image[6], such as finding the size of $J \times K$ of the subsidiary image mode $w(x, y)$ in $M \times N$ image $f(x, y)$, the correlation of $f$ and $w$ can be expressed as equation (1):

$$
c(x, y)=\sum_{s=0}^{K} \sum_{t=0}^{J} w(x, t) f(x+s, y+t)
$$

But the above equation is sensitive to the gray value of $f$ and $w$ when calculating the response, which is a defect, this paper normalized the vector modulus value to resolve this problem[7], an improved correlation calculation formula for the match as below (2):

$$
r(x, y)=\frac{\sum_{s=0}^{K} \sum_{t=0}^{J} w(x, t) f(x+s, y+t)}{\left[\sum_{s=0}^{K} \sum_{t=0}^{J} w^{2}(s, t) \cdot \sum_{s=0}^{K} \sum_{t=0}^{J} f^{2}(x+s, y+t)\right]^{1 / 2}}
$$

Intercept marker shape temple, using artificial marker method, first calibrate two lateral points in the region need to be extracted, then after the identification of calibration point, according to the requirements to set the length and width of the extraction range, or use more than one calibration points to calibrate extract range directly, and then calculates the coordinates of the calibration point then extracts the image of calibration area.

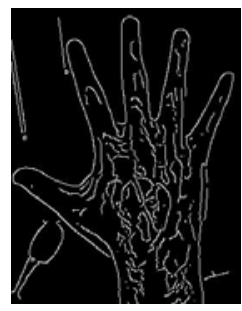

Fig.2 Canny algorithm extract edge

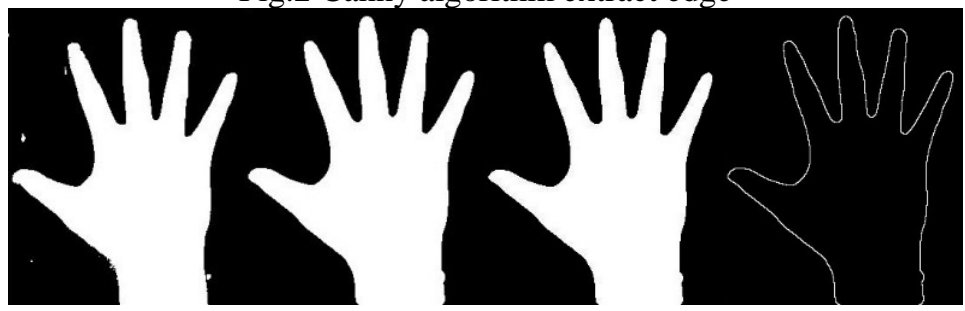

Fig.3 a.Binary image

b. Image after filtering

c. After corroding

d. Opisthenarcontour

Improve ROI extraction algorithm in reference [5], so that an image of outstretched hand can be 
extracted. The effective area of hand vein is below the second and fourth finger web. To extract the image, position the coordinates of two finger webs firstly.

Step one, extracting the contour of opisthenar. Due to the working environment of Kinect, it determines the image background contains more noise, when using common Canny algorithm to extract edge contour, the background will have a greater impact on the final contour[8], as shown in Fig.2. This paper adopts the following method to extract the opisthenar contour:

A. binary process the image, to achieve Fig. 3 a;

B. $\quad$ median filtering Fig. 3 to obtain Fig.3 b;

C. $\quad$ use $3 \times 3$ symmetric structural elements to corrode Fig. 3 b to obtain Fig. 3 c;

D. Fig.3 c subtracting the filtered image Fig.3 b, to get the outline of Fig.3 d, compared to

Fig.2, the outline is very clear, the background noise is also completely eliminated.

The second step, determining the center position $\left(O_{x}, O_{y}\right)$ of opisthenar, and use the equation (3) to calculate the distance from contour points of the opisthenar to the center position[9]:

$$
S=\sqrt{\left(O_{x}-X(i)\right)^{2}+\left(O_{y}-Y(i)\right)^{2}}, i=1,2,3 \ldots n
$$

Where $X(i), Y(i)$ is the abscissa and ordinate of the $i$-th contour point; $\mathrm{n}$ is the sum of contour points in the opisthenar contour map.

The third step, determine the coordinates of the finger web. Search the three points whose distances are shorter, and sort by abscissa, get the second and fourth finger web coordinates $\left(P_{x 2}\right.$, $\left.P_{y 2}\right)$ and $\left(P_{x 4}, P_{y 4}\right)$.

The fourth step, rotating the image by using the equation (4), to ensure the abscissas of two finger webs are equal and at the same horizon.

$$
\theta=\arctan \left(\left(P_{x 2}-P_{x 4}\right) /\left(P_{y 2}-P_{y 4}\right)\right) \times 180 / \pi
$$

Where $\theta$ represents the angle of rotation.

The fifth step, extract the effective area of the square. To be able to fully extract the vein image, 1.2 times the second and fourth finger web distance, determine the coordinates of the 4 apexes of the square, then extract the image.

After comparison, the advantages of methods based on relevant template matching are, any marked area can be extracted (less than 3 calibration points), and do not need the image binarization processing; disadvantages are inevitable error of artificial mark, the similarity of label graphics and background graphics leads to error calibration, large scale of computation[10]. Improved ROI extraction algorithm is easy to use, strong adaptability, but acquired image need to be adequately addressed, make sure that the opisthenar contour points are not influenced by the background image. Since the extract object of this paper is limited to the opisthenar image, and improved extraction algorithm can be accurately extracted under most conditions, so this paper use the improved ROI extraction algorithm.

\section{Vein Image Processing}

Extracted vein image have rarely effective pixels, using threshold segmentation method to highlight effective vein in the vein image extracted, compared to other methods of the threshold segmentation method is intuitive and easy to implement, are widely applied in applications focused on operational efficiency. In this paper, a large local threshold method is used in image processing, segment the original image into a number of sub-images, then determine the optimal threshold value respectively. Principle of using iterative method to calculate the optimal segmentation threshold of the sub-images is shown as below:

$f(x, y), Z(i, j)$ represents the gray value of image point $(i, j)$, denote $N(i, j)$ as the weight coefficient of point $(i, j)$.

A. obtain the initial threshold $T_{0}$ of the maximum and minimum gray value $Z_{k}$ and $Z_{0}$ in the originalpicture.

$$
T_{0}=\left(Z_{0}+Z_{k}\right) / 2
$$


B. divide image into two parts, target and background according to the thresholds $T_{k}$ obtained, calculate the average gray value $Z_{0}, Z_{B}$ respectively.

C. findthe new threshold value $T_{k}+1$.

$$
Z_{0}=\frac{\sum_{z(i, j)<T_{k}} z(i, j) \times N(i, j)}{\sum_{z(i, j)<T} N(i, j)}, Z_{B}=\frac{\sum_{z(i, j)>T_{k}} z(i, j) \times N(i, j)}{\sum_{z(i, j)<T} N(i, j)}
$$

$$
T_{k+1}=\frac{Z_{0}+Z_{B}}{2}
$$

D. if $T_{k}=T_{k+1}$, then ends. Otherwise, $k+1 \rightarrow k$, turn to step two.

E. after the fourth step, the resulted $T_{k}$ is the optimal threshold value.

In using local threshold method to process, the size of the average filter window needs to be selected, while the bigger the window the more significant the filtering is, but the more details are lost, the smaller the window the more information is retained and the not more obvious the filtering is. The average size of the filter window need to be determined based on imaging object and its conditions, can be selected by virtue of experience, aimed at the imaging conditions through repeated experiments in this paper, the test window size is "11" when the effect is the best. Then median filtering again, to obtain the final image.

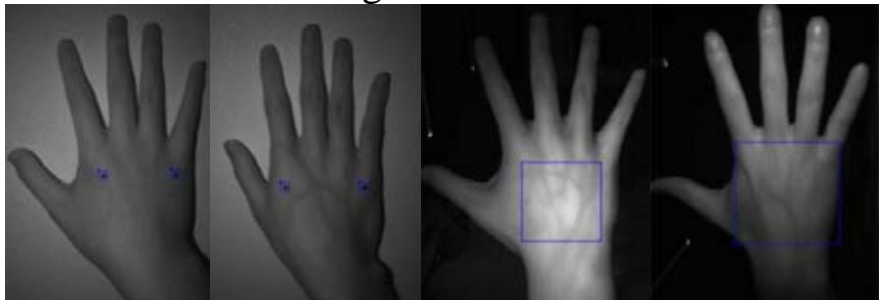

Fig.4Template matching recognition of different opisthenar markers andROI recognition of different effective area

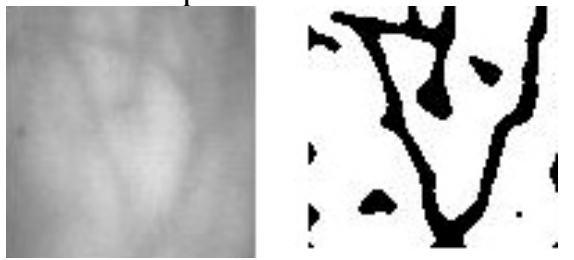

Fig.5The processed image of effective area

\section{Results And Discussion}

Through the collection of different opisthenar image by Kinect, validating the algorithm using Matlab, the calibration result based on relevant temple matching is shown in Fig.4, the effective area of the image extracted by ROI is shown at last two images in Fig.4, it can be seen that reliable area can be to identified validly. Fig. 5 shows the extraction image after processed, the exact location is visible where the vein is.

\section{Conclusion}

This paper presents a Kinect-based acquisition method of hand vein, also introduces template matching based on relevance and improved two image extraction methods of reference[5]. Firstly ,use Kinect to capture images; Secondly, extract the effective area of the hand vein, images can be extracted directly when using the template matching method, but the opisthenar contour needs to be extracted and the distance from each contour point to the center of opisthenar calculated before when choosing the second extraction method, determine the location of the second and fourth finger web, then intercept effective area; finally, threshold preprocess the obtain images, then get final image by median filtering. After verification by Matlab, it proves that this scheme contains the advantages of easier to achieving, accurate extracting and processing effect good. 
However, as the infrared device used is Kinect's own whose infrared light powerful is limited, when shooting the user with thick fat layer, the image quality is not high, external infrared light source can be added later, or enhancing an image processing, so that further optimizing the quality of extracted effective image.

\section{Acknowledgements}

This work was financially supported by the Chinese National Natural Science Foundation (61402063) and Chongqing University Outstanding Achievement Transformation Project (KJZH14213).

\section{References}

[1] Zhu Tao, Jing Guodong, Lu Lifu, The brief summary of Kinect application and its development prospect, Modern computer: next half month edition, Vol. 4(2013), pp. 8-11.

[2] Ni Xuxiang, Gao Shijie, Wei Keyu etc., Hand vein imaging based on partition multiple exposures,Photon Journal, Vol.42(2013), p. 1534-1538.

[3] Yu Tao. Kinect application development: use the most natural way to dialogue with the machine, Beijing(2013), p. 32-33.

[4] Chen Yan, NiuYanxiong, Tang Fang etc, Experimental study on the thermal effect of skin tissue irradiated by 1319nm laser,Photon Journal, Vol. 38(2009), p. 1259-1264.

[5] Miura N, Nagasaka A, Miyatake T, Feature extraction offinger-vein patterns based on repeated line tracking and its application to personal identification, Vol. 15(2004), p. 194-203.

[6] Chen Long, HouPuhua, Algorithm of vehicle license plate extraction based on ROI under complex environment,Information technology, (2013), p. 95-98.

[7] Li Qiang, Zhang Ba, A fast matching algorithm based on image gray value,Information technology, (2013),p. 95-98.

[8] Zhang Heng, Gao Min, Xu Chao, Image segmentation algorithm of fusion threshold method and edge extraction, Journal of The Academy of Armored Forces Engineering, Vol. 28(2014) , p. 73-86.

[9] Wu Zuoling, Chen Xiong, Zheng Yingjie, An image recognition algorithm for near infrared back of hand,Microelectronics and computer, Vol. 27(2010), p. 114-116, Oct.

[10]Zhang Zheng, Ni Hongxia, Yuan Chunmiao etc. Proficient in Matlab digital image processing and recognition, Beijing, 2013, p. 338-339. 